\title{
The Cross-Sectional Evaluation of the Use of Artemisinin-Based Combination Therapy for Treatment of Malaria Infection at a Tertiary Hospital in Nigeria
}

\author{
Roland Nnaemeka Okoro ${ }^{1}{ }^{1}$ and Muslim Olakunle Jamiu ${ }^{2}$ \\ ${ }^{1}$ Department of Clinical Pharmacy and Pharmacy Administration, Faculty of Pharmacy, University of Maiduguri, Maiduguri, Nigeria \\ ${ }^{2}$ Department of Clinical Pharmacy and Pharmacy Practice, Faculty of Pharmaceutical Sciences, University of Ilorin, Ilorin, Nigeria \\ Correspondence should be addressed to Roland Nnaemeka Okoro; orolandn@gmail.com
}

Received 27 November 2017; Accepted 25 March 2018; Published 16 May 2018

Academic Editor: Sukla Biswas

Copyright @ 2018 Roland Nnaemeka Okoro and Muslim Olakunle Jamiu. This is an open access article distributed under the Creative Commons Attribution License, which permits unrestricted use, distribution, and reproduction in any medium, provided the original work is properly cited.

\begin{abstract}
In 2005, Nigeria changed its antimalarial drug policy to Artemisinin-based Combination Therapies (ACTs) for the treatment of malaria infection, and it is imperative for prescribers to strictly comply with this guideline to harmonize malaria management practices within the country. This study aims to evaluate prescribers' adherence with the National Antimalarial Treatment Guideline (NATG) in the treatment of malaria infections and to describe the determinants of antimalarial drugs coprescription with antibiotics at a tertiary hospital in Nigeria. A cross-sectional, retrospective study of antimalarial drug prescriptions of one-year period of 2013 was conducted. A simple method for assessing the quality of drug prescribing (DU90\%) was adopted. Logistic regression was used to predict antimalarial drugs coprescription with antibiotics. Overall, $95.8 \%$ of the total prescriptions contained ACTs, out of which $80.8 \%$ were Artemether/Lumefantrine. However, adherence to NATG was $88.2 \%$ with an adjusted value of $100.0 \%$. Age was the only predictor for antimalarial drugs coprescription with antibiotics. This study showed high concordance with NATG at the studied hospital. Age less than 5 years is a significant risk factor for antimalarial drugs coprescription with antibiotics.
\end{abstract}

\section{Introduction}

Malaria remains the most common public health problem in Nigeria where it accounts for more cases and deaths than any other country in the world. Malaria is a risk for majority of Nigeria's population with an estimated 100 million malaria cases with over 300,000 deaths per year in Nigeria [1]. Children under 5 years of age are the most vulnerable group and account for significant malaria deaths [2]. The aim of proper malaria diagnosis and treatment is to cut the disease burden, death, and socioeconomic losses. The use of national guidelines for malaria diagnosis and treatment is paramount to achieving this goal. The antimalarial drug policy was formulated to promote rational use of antimalarial drugs in order to reduce the development of drug resistance.

Following a period of continuous increase in resistance of Plasmodium falciparum against the commonly used antimalarial drugs such as chloroquine, and SulphadoxinePyrimethamine (SP), the new artemisinin-based combination therapy (ACT) was introduced in 2005 with ArtemetherLumefantrine (AL) as first-line treatment for uncomplicated malaria and Artesunate + Amodiaquine (copackaged) as alternative $[3,4]$. However, the meta-analysis conducted in 2014 reported that the prevalence of use of ACT in the public sector in Nigeria was 76\% [5].

To meet the goal of universal access to right interventions for all populations at risk of malaria, it is required that the proper clinical investigation is conducted prior to treatment with effective antimalarial drugs [4]. However, one of the main obstacles to health care access is the direct out-ofpocket payment form of health care financing which occurs in most sub-Saharan African countries [6]. In a bid to promote access to quality health care services, Nigerian government introduced National Health Insurance Scheme (NHIS) in 
2005. Evidence shows that the National Health Insurance policy has led to an increase in utilization of medicines [7]. In spite of this, as at 2016 the scheme has covered only about 4 percent of total Nigerian population [8].

Appropriate treatment of malaria and the correct use of antimalarial drugs are needed in order to achieve Nigerian's goal of preelimination and reducing malaria related death to zero by 2020 [9]. Drug use studies are carried out to find out associated problems with drug use since adherence of health providers and patients to recommendations of a treatment policy is key to the overall success of such guidelines. Drug use studies could unravel drug use problems and give feedback to the prescriber to create awareness on rational use of drugs [10]. As such, antimalarial drug use studies are important in an effort to redress the impact of malaria as it will make sure that care providers in the health insurance scheme are working in concert with national strategy.

In Nigeria today, there is paucity of data on the implementation of this new antimalarial policy in the NHIS. An NHIS antimalarial prescription audit has been done in the South Western part of the country [11]. To our knowledge, no antimalarial drug use study has been conducted in NHIS in the South Eastern Nigeria, hence the need for this study. It is necessary to survey antimalarial drug use among insured patients at a tertiary hospital in this part of the country to offer insights into provider's antimalarial drugs prescribing practices and to gather baseline information that could serve as a basis for designing appropriate interventions and policies to improve antimalarial drugs use within the scheme. The aims of this study were to evaluate prescribers' adherence with the National Antimalarial Treatment Guideline in the treatment of malaria infections and to assess the determinants of antimalarial drugs coprescription with antibiotics in the NHIS at tertiary hospital in Nigeria.

\section{Method}

2.1. Study Site and Design. This was a descriptive, crosssectional, retrospective study of prescriptions purposively carried out among NHIS outpatients at the University of Nigeria Teaching Hospital (UNTH), Enugu. NHIS outpatients are seen by medical doctors from various specialties. These patients access their NHIS approved drugs with a $10 \%$ copayment only from the NHIS outpatients' pharmacy unit. UNTH is a tertiary health care facility of about 500-bed capacity with staff made up of professionals and nonprofessionals. It serves as the teaching hospital for the faculty of medicine of the University of Nigeria and is a participating Health Care Provider (HCP) on the insurance scheme.

2.2. Sampling. This comprised all NHIS outpatients' prescriptions that contained at least one antimalarial drug filled from January to December 2013. Prescriptions from antenatal clinic were excluded due to the use of SP for Intermittent Preventive Therapy (IPT) in pregnancy.

Large sample size which exceeded the minimum of 100 suggested by WHO was employed in order to enhance the reliability of the results since only one health facility was used for the study [10]. Systematic sampling was used to select the prescriptions. Based on the total number of 6667 prescriptions containing at least one antimalarial drug after excluding those from antenatal clinic, a sampling interval of 20 was calculated and simple balloting was used for the first pick. At the end, a total number of three hundred and thirtythree (333) prescriptions containing at least one antimalarial drug were selected for the study.

2.3. Data Collection. The modified World Health Organization (WHO) prescribing indicator form was used to extract the following data: age; sex; month of prescription; the names of antimalarial drugs prescribed; number of drugs prescribed; number of drugs dispensed; number of drugs prescribed from Essential Drug List (EDL) [12]; number of drugs prescribed by generic name; encounter with an antibiotic prescribed; and encounter with an injection prescribed. These data were later entered into Microsoft Excel (Microsoft Corporation, 2007).

2.4. Data Management and Analysis. Abstracted information was later keyed into Statistical Package for Social Sciences (SPSS, version 21, Chicago, USA) coded for data analysis. Raw data were double-checked with soft data for consistency. The WHO drug use indicators investigated in this study were the average number of drugs per encounter, percentage encounter with an antibiotic, percentage encounter with an injection, number of drugs prescribed by generic name, and the number of drugs prescribed from NHIS EDL described elsewhere [10]. Drug use metric employed was number of prescriptions and drug use pattern was expressed in terms of Drug Utilization Ninety Percent (DU90\%). The DU90\% segment reflects the number of drugs that account for $90 \%$ of drug prescriptions. Adherence with the regimens listed in the National Antimalarial Treatment Guideline (NATG) was assessed [13]. Adherence was defined as number of prescribed regimens recommended from the NATG divided by number of regimens in the DU90\% segment, expressed in percentage. Adjusted adherence represents the percentage of all regimens listed in the NATG in addition to other ACTs not listed in NATG. Logistic regression was used to model the predictors on the outcome variable (antimalarial drug coprescription with antibiotics). Statistical significance was set as a $p$ value less than $0.05(p<0.05)$.

2.5. Ethics. Permission to conduct the study was sought from the hospital management and ethical clearance was obtained from the Research and Ethics Committee of the UNTH.

\section{Results}

3.1. Patients Baseline Demographic Characteristics. Demographic information of the study patients is presented in Table 1. In total, 333 prescriptions containing antimalarial drugs were reviewed. It was found that more than onehalf $(69.4 \%)$ of the prescriptions were written for female patients. About $54.0 \%$ of the patients were older than 18 years (Table 1). 
TABle 1: Demographic characteristics of patients prescribed antimalarial drugs $(N=333)$.

\begin{tabular}{lc}
\hline Variable & $n(\%)$ \\
\hline Gender & \\
Female & $231(69.4)$ \\
Male & $104(30.6)$ \\
Age group (years) & \\
$<5$ & $21(6.3)$ \\
$5-11$ & $24(7.2)$ \\
$12-18$ & $11(3.3)$ \\
$>18$ & $179(53.8)$ \\
Not indicated & $98(29.4)$ \\
Treatment period & \\
First quarter & $80(24.0)$ \\
Second quarter & $125(37.5)$ \\
Third quarter & $118(35.4)$ \\
Fourth quarter & $10(3.0)$ \\
\hline
\end{tabular}

A quarter refers to one-fourth of a year (a period of 3 months).

3.2. Prescription Pattern of Antimalarial Drugs. Table 2 shows that, among the ACTs and nonartemisinin monotherapy, AL and Proguanil were the most prescribed antimalarial drugs, respectively. On evaluation of the artemisinin monotherapy prescribed, it was found that one-half (Artemether 16.7\% and Arteether $33.3 \%$ ) was given as injectables.

\subsection{Drug Utilization Ninety Percent (DU90\%) of Antimalarial} Drug Regimen. Figure 1 shows the antimalarial regimens within the drug utilization (DU90\%) segment. Only three drugs appeared in the DU90\% segment with a high adherence of $88.2 \%$ to NATG and adjusted adherence of $100.0 \%$.

3.4. Prescribing Indicators. Table 3 shows the drug use indicators. The total number of drugs prescribed was 1,585 with an average number of drugs per prescription of 4.8. The maximum number of drugs prescribed per prescription was 11 , representing $0.6 \%$ of all prescriptions. The median number of drugs per prescription was 5 .

3.5. Determinants of Antimalarial Drugs Coprescription with Antibiotics. Table 4 summarizes the results of predictors for antimalarial drugs coprescription with antibiotics. Age was the only significant predictor for antimalarial drugs coprescription with antibiotics.

\section{Discussion}

This study revealed high prevalence of use of ACTs especially $\mathrm{AL}$ for the treatment of malaria infections among insured patients. AL is the antimalarial drug of choice for the treatment of uncomplicated malaria in Nigeria due to its demonstrated efficacy. The results of the drug efficacy trials carried out in the all the geopolitical regions of the country in 2004 found AL to be highly efficacious and thus suitable for use in the treatment of uncomplicated $P$. falciparum malaria

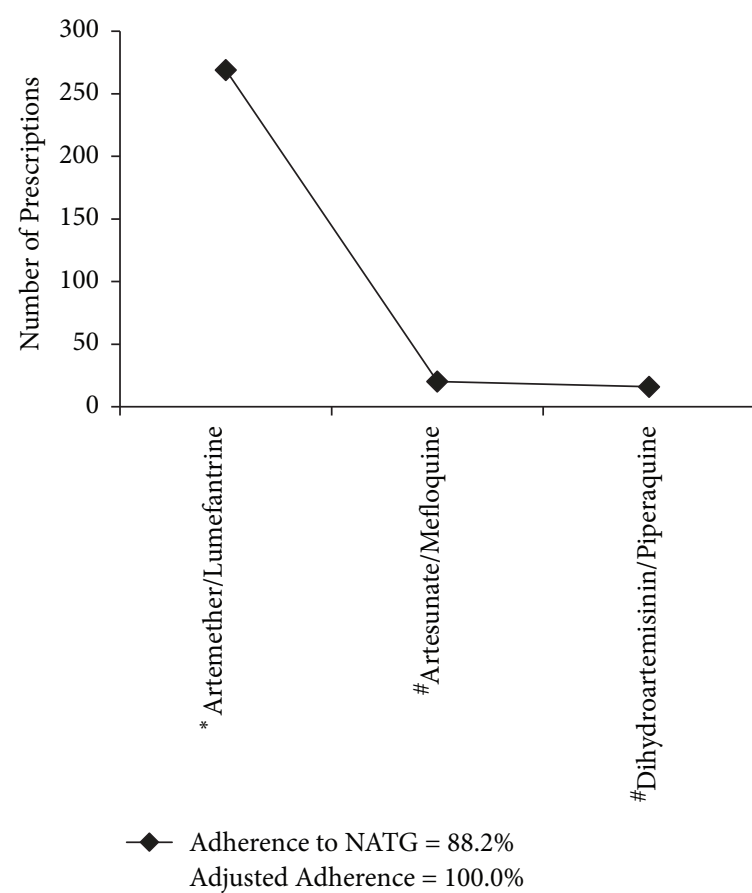

FIGURE 1: Drug utilization ninety percent (DU90\%) of antimalarials. ${ }^{*}$ Antimalarial regimen listed in the NATG. ${ }^{*}$ Antimalarial regimens that are not listed in the NATG, but can be referred to as "recommended" treatment as they contain an artemisinin derivative and partner drug. Adherence: Based on antimalarial regimens listed in the NATG. Adjusted Adherence: Based on all ACTs.

in the country [3]. This finding is consistent with the findings of other Nigerian studies [14-17], but inconsistent with a previous NHIS study done in another part of the country [11]. Secondly, there was high concordance with NATG at the study setting which is commendable. Interestingly, only three antimalarial drug regimens made it to the DU90\% segment. This result is suggestive of high quality prescribing of antimalarial drugs in the setting studied. It has been found that the use of larger number of choices of therapy results in a decreased rational clinical decision-making process [18]. Therefore, high quality prescribing is associated with the use of a relatively limited number of pharmaceutical products [19]. Besides high quality prescribing, our study recorded a higher adherence to NATG compared to a non-NHIS study that reported lower levels of $66.7 \%$ in secondary hospital and $38.5 \%$ with adjusted adherence of $69.2 \%$ in a tertiary hospital. Our finding demonstrates the usefulness of NHIS as a tool towards realizing the goal of eliminating malaria related morbidity and mortality in Nigeria by 2020.

Analysis of drug prescribing indicators revealed high average number of drugs prescribed per encounter which indicates occurrence of polypharmacy. This result is comparable with the values of 3.4, 3.8, and 4.1 got from earlier NHIS studies in tertiary hospitals in South Western, North Eastern, and North Western Nigeria [7, 20, 21]. A plausible reason for this polypharmacy in NHIS at the tertiary hospitals might be reasonable in some cases due to high prevalence of comorbidity, while unjustifiable ones could be attributed 
TABLE 2: Prescription pattern of antimalarial drugs $(N=333)$.

\begin{tabular}{lc}
\hline Antimalarial drug regimens prescribed & $n(\%)$ \\
\hline Non-artemisinin monotherapies (NAMTs) & $8(2.4)$ \\
Proguanil & $5(1.5)$ \\
Sulphadoxine-Pyrimethamine & $2(0.6)$ \\
Amodiaquine & $1(0.3)$ \\
Artemisinin monotherapies (AMTs) & $6(1.8)$ \\
Artesunate & $2(0.6)$ \\
Arteether & $2(0.6)$ \\
Artemether & $1(0.3)$ \\
Dihydroartemisinin & $1(0.3)$ \\
Artemisinin combination therapies (ACTs) & $319(95.8)$ \\
Artemether/Lumefantrine & $269(80.8)$ \\
Artesunate/Mefloquine & $20(6.0)$ \\
Dihydroartemisinin/Piperaquine & $16(4.8)$ \\
Artesunate/Amodiaquine & $8(2.4)$ \\
Artesunate/Piperaquine & $4(1.2)$ \\
Artemether/Lumefantrine + Sulphadoxine-Pyrimethamine & $1(0.3)$ \\
Artesunate/Sulphadoxine-Pyrimethamine + Proguanil & $1(0.3)$ \\
\hline
\end{tabular}

TABLE 3: Drug prescribing indicators.

\begin{tabular}{lc}
\hline Variable & Value \\
\hline Average number of drugs prescribed per encounter (mean \pm SD) & $4.8 \pm 1.8$ \\
Percentage encounter with an antibiotic (\%) & 37.2 \\
Percentage encounter with an injection (\%) & 1.5 \\
Percentage of drugs prescribed by generic name (\%) & 49.3 \\
Percentage of drugs prescribed from EDL (\%) & 63.0 \\
Percentage drugs dispensed (\%) & 91.8 \\
\hline
\end{tabular}

TABLE 4: Predictor variables for antimalarial drug coprescription with antibiotics.

\begin{tabular}{lccc}
\hline Variable & Adjusted odds ratio & 95\% CI & \\
\hline Age group (years) & & & \\
$<5$ years & Reference & $0.04-0.61$ & $0.04-1.03$ \\
$5-11$ years & 0.16 & $0.11-0.75$ & 0.055 \\
$12-18$ years & 0.20 & & \\
$>18$ years & 0.29 & & \\
Gender & & $0.52-1.42$ \\
Female & Reference & & \\
Male & 0.86 & & \\
Treatment period & & $0.48-1.58$ \\
First quarter & Reference & $0.51-1.72$ \\
Second quarter & 0.87 & $0.44-6.76$ \\
Third quarter & 0.94 & 0.551 \\
Fourth quarter & 1.72 & 0.650 \\
\hline
\end{tabular}

to patients' demand for drugs and the fact that NHIS is a subsidized scheme in which patients pay only $10 \%$ of the medication fees as copayment. The current study also revealed low generic prescribing which is congruent with other Nigerian studies done in the NHIS $[7,20,21]$. Low value was found in these studies despite the fact that Nigerian National Health
Insurance Policy, published in 2005, prescribed that purchase and prescribing of drugs in the NHIS shall be by generic names only. The implications of low generic use are primarily the wastage of scarce health resources and a decrease in access to pharmaceuticals because of affordability barrier to patients if medicine out of stock occurs in the NHIS. 
However, evaluation of encounters with antibiotics revealed a higher value of $37.2 \%$ as against the $\mathrm{WHO}$ reference range of $20.0 \%-26.8 \%$ [22]. Other NHIS studies have also reported values above WHO reference range $[7,20]$. Concomitant prescription of antimalarial drugs and antibiotics occurs usually to take care of any potential coinfection implying that the prescriber is not too certain about the real diagnosis, or to prevent undetectable infection showing up [23]. Overuse of antibiotics leads to emergence of drug resistance microorganisms and increased side effects and cost of healthcare. Therefore, this finding serves as a clarion call for improved diagnosis of nonmalarial fever and to physicians to prescribe antibiotics with caution to cut these negative health outcomes.

The low number of drugs prescribed from the EDL was due to nonrevision of NHIS EDL 2005 edition for almost a decade. Implementation of this EDL after 8 years constrained the prescribers in the scheme, thereby forcing them to prescribe some drugs not listed in the NHIS EDL that were considered effective based on clinical judgment to meet the desired health outcomes for the patients. The concept of essential drugs incorporates the need to regularly delete obsolete medicines and add newer more effective ones to reflect new treatment options and changing therapeutic needs among others [24].

The low use of injections and high amount of medicine dispensed were commendable. Use of injections is associated with adverse events and increased cost. On the other hand, high number of medicines dispensed reported by this study shows that the hospital procured some medicines outside the NHIS EDL to decrease patients out-of-pocket expenses on medicines due to the restriction imposed by the outdated NHIS EDL.

Furthermore, this study assessed the factors associated with antimalarial drugs coprescription with antibiotics. Predictor found to be associated with the risk of being coprescribed antimalarial drugs with antibiotics was age. Children under five years of age were much more likely to be coprescribed antimalarial drugs and antibiotics than those aged 5 years and more. Similar findings were reported in South Eastern and South Western Nigeria [17, 25], Ghana [23], and India [26]. This might be due to paediatrics having high risk to suffer from recurrent infections of other systems such as the respiratory tract (cough) and gastrointestinal system (diarrhoea).

The limitations of this study include the retrospective collection of prescription data. This made it impossible for parasitological and patient clinical results to be collected. Therefore, use of drug could not be linked with the clinical decisions that informed the prescriptions. However, only prescription data was used to describe quality indicator index of drug prescribing: DU90\%. Due to time lag between data collection and publishing of findings, the study findings may not reflect the current antimalarial drug prescribing practice of the setting studied. Lastly, the use of only one centre for the study restricts the generalization of the findings.

\section{Conclusions}

This study showed a higher use of artemisinin-based combination therapy and high concordance with National
Antimalarial Treatment Guideline for the treatment of malaria infection. Based on this result, policy makers can consider addressing Universal Health Coverage (UHC) as health priority for Nigerian citizens as part of national malaria elimination strategy. Age less than 5 years is a significant risk factor for antimalarial drugs coprescription with antibiotics We recommend that prescribers at the study setting should conduct appropriate test to confirm bacterial infections before prescribing antibiotics especially for this vulnerable age group.

\section{Conflicts of Interest}

The authors declare that they have no conflicts of interest to disclose regarding the publication of this paper.

\section{Supplementary Materials}

Additional file (xls) containing antimalarial drug information and drug prescribing indicators data. (Supplementary Materials)

\section{References}

[1] Nigeria Malaria Fact Sheet, https://photos.state.gov/libraries/ nigeria/231771/Public/December-MalariaFactSheet2.pdf.

[2] WHO Global Malaria Programme, World Malaria Report 2014.

[3] Federal Republic of Nigeria: National Antimalarial Treatment Policy, FMOH, National malaria and Vector Control Division, Abuja, Nigeria, 2005.

[4] Federal Ministry of Health, National Malaria Control Programme, Abuja, Nigeria. Strategic Plan 2009-2013, 2008, Federal Ministry of Health, National Malaria Control Programme.

[5] A. M. Yakasai, M. Hamza, M. M. Dalhat et al., "Adherence to Artemisinin-Based Combination Therapy for the Treatment of Uncomplicated Malaria: A Systematic Review and MetaAnalysis," Journal of Tropical Medicine, vol. 2015, Article ID 189232, 2015.

[6] D. B. Evans and C. Etienne, "Health systems financing and the path to universal coverage," Bulletin of the World Health Organization, vol. 88, no. 6, pp. 402-403, 2010.

[7] J. Fadare, A. Adeoti, F. Aina, O. Solomon, and J. Ijalana, "The influence of health insurance scheme on the drug prescribing pattern in a Nigerian tertiary healthcare facility," Nigerian Medical Journal, vol. 56, no. 5, p. 344, 2015.

[8] P. Duru, Only 4\% of Nigerians covered by NHIS, Vanguard Newspaper, http://www.vanguardngr.com/2016/12/4-nigerians-covered-nhis/.

[9] Federal Ministry of Health, "National Malaria Control Programme, Abuja, Nigeria," Strategic Plan 2014-2020, 2015.

[10] World Health Organization. How to investigate drug use in health facilities: selected drug indicators, Action Programme on Essential Drugs (WHO/DAP/(93.11) Geneva, 1993.

[11] A. E. Joda and M. O. Ologunagba, "Antimalarial prescribing under the National Health Insurance Scheme (NHIS)," West African Journal of Pharmacy, vol. 25, no. 1, pp. 97-105, 2014.

[12] NHIS, Healthcare providers service price list, 2005, http:// dhmlnigeria.com/downloads/NHIS_drugs_pricelist_2011.pdf.

[13] Federal Republic of Nigeria: National guidelines for diagnosis and treatment of malaria, FMOH; National malaria and Vector Control Division, Abuja, Nigeria, 2007. 
[14] N. U. Igboeli, C. V. Ukwe, and O. I. Ekwunife, "Increasing use of artemisinin-based combination therapy for Treatment of malaria infection in Nigerian hospitals," Pharmacy Practice, vol. 8, no. 4, pp. 243-249, 2010.

[15] S. A. Hudu, A. O. Jimoh, K. Abubakar, and A. Bello, "Common anti-malarial drug prescription and patient affordability in sokoto Nigeria," International Journal of Pharmacy and Pharmaceutical Sciences, vol. 5, no. 2, pp. 428-431, 2013.

[16] I. O. Ishola, I. A. Oreagba, S. O. Olayemi, and R. Gbadamosi, "Assessment of treatment pattern of uncomplicated malaria in peadiatric patients attending a teaching hospital in northwest Nigeria," Journal of Applied Pharmaceutical Science, vol. 1, no. 5, pp. 177-1781, 2011.

[17] C. C. Ezenduka, M. J. Okonta, and C. O. Esimone, "Adherence to treatment guidelines for uncomplicated malaria at two public health facilities in Nigeria; implications for the 'test and treat' policy of malaria case management," Journal of Pharmaceutical Policy and Practice, vol. 7, no. 1, pp. 1-10, 2014.

[18] V. Chinburapa, L. N. Larson, M. Brucks, J. Draugalis, J. L. Bootman, and C. P. Puto, "Physician prescribing decisions: The effects of situational involvement and task complexity on information acquisition and decision making," Social Science \& Medicine, vol. 36, no. 11, pp. 1473-1482, 1993.

[19] U. Bergman, C. Popa, Y. Tomson et al., "Drug utilization 90\% A simple method for assessing the quality of drug prescribing," European Journal of Clinical Pharmacology, vol. 54, no. 2, pp. 113-118, 1998.

[20] R. N. Okoro and B. G. Shekari, "Physicians/ drug prescribing patterns at the national health insurance scheme unit of a teaching hospital in the North Eastern Nigeria," Archives of Pharmacy Practice, vol. 4, no. 1, p. 3, 2013.

[21] S. N. Abdu-Aguye, A. Haruna, A. Shehu, and K. S. Labaran, "An assessment of antimicrobial prescribing at a tertiary hospital in north-western Nigeria," The African Journal of Pharmacology and Therapeutics, vol. 5, pp. 229-234, 2016.

[22] A. Isah, R. Laing, J. Quick et al., “The Development of Reference Values for the WHO Health Facility Core Prescribing Indicators," West African Journal of Pharmacology and Drug Research, vol. 18, no. 1, 2001.

[23] A. N. O. Dodoo, C. Fogg, A. Asiimwe et al., "Pattern of drug utilization for treatment of uncomplicated malaria in urban Ghana following national treatment policy change to artemisinin-combination therapy," Malaria Journal, vol. 8, no. 1, article no. 2, 2009.

[24] “Essential Drugs Programme (EDP)," http://www.health.gov .za/index.php/essential-drugs-programme-edp.

[25] J. Fadare, O. Olatunya, O. Oluwayemi, and O. Ogundare, "Drug prescribing pattern for under-fives in a paediatric clinic in South-Western Nigeria," Ethiopian Journal of Health Sciences, vol. 25, no. 1, pp. 73-78, 2015.

[26] J. R. Torvi and S. Dambal, "Drug prescription pattern in paediatric out patient clinic in a tertiary hospital," Current Pediatric Research, vol. 15, no. 2, pp. 77-80, 2011. 


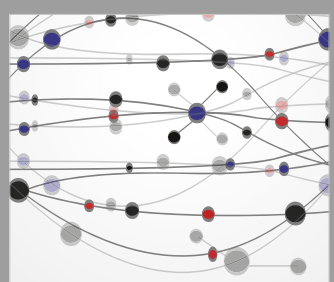

The Scientific World Journal
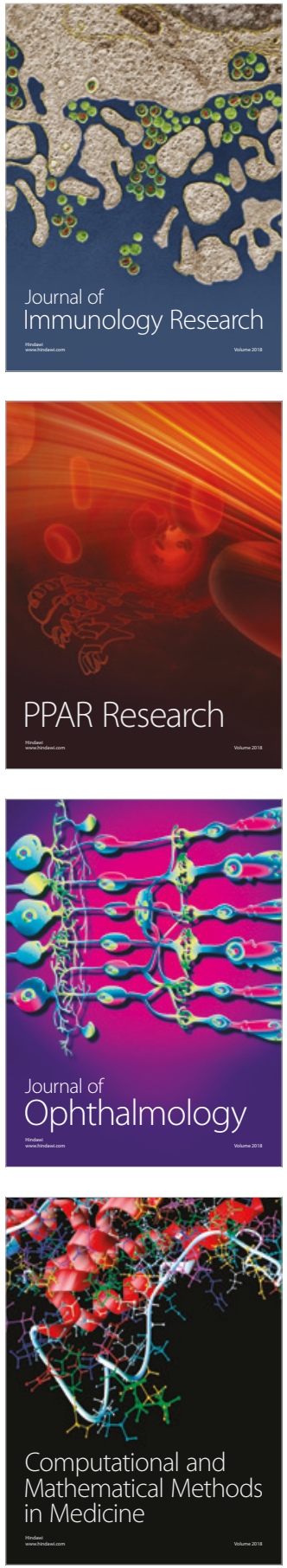

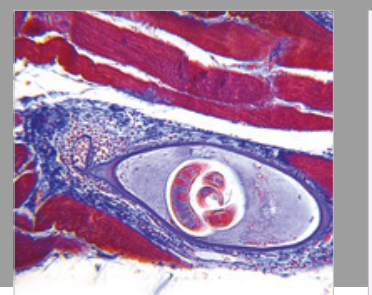

Gastroenterology Research and Practice

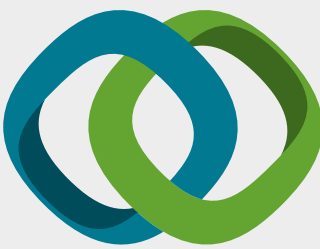

\section{Hindawi}

Submit your manuscripts at

www.hindawi.com
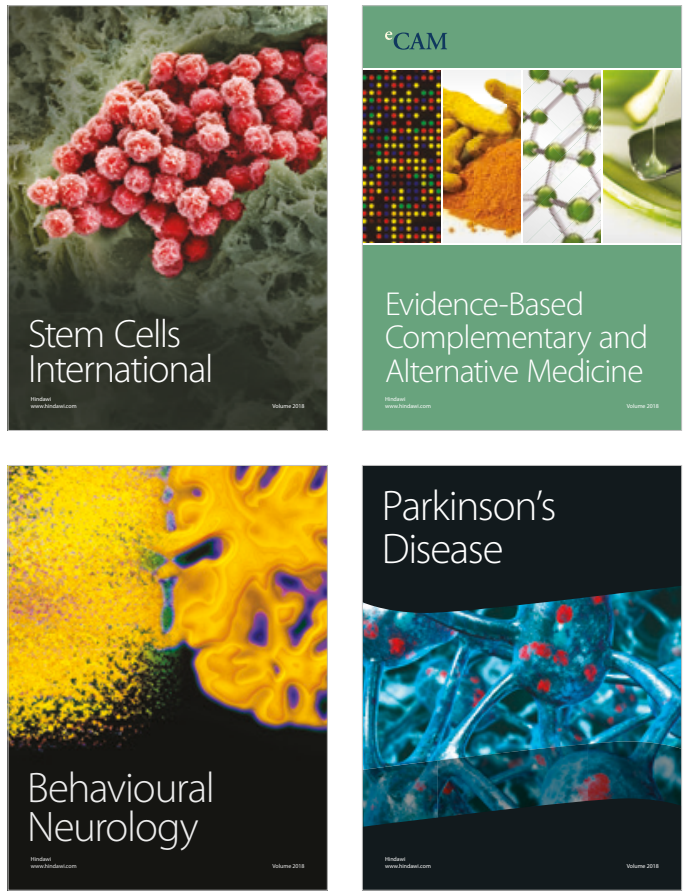

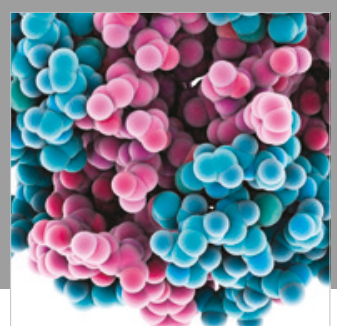

ournal of

Diabetes Research

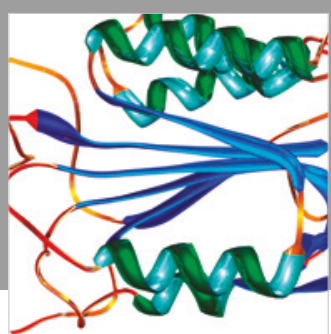

Disease Markers
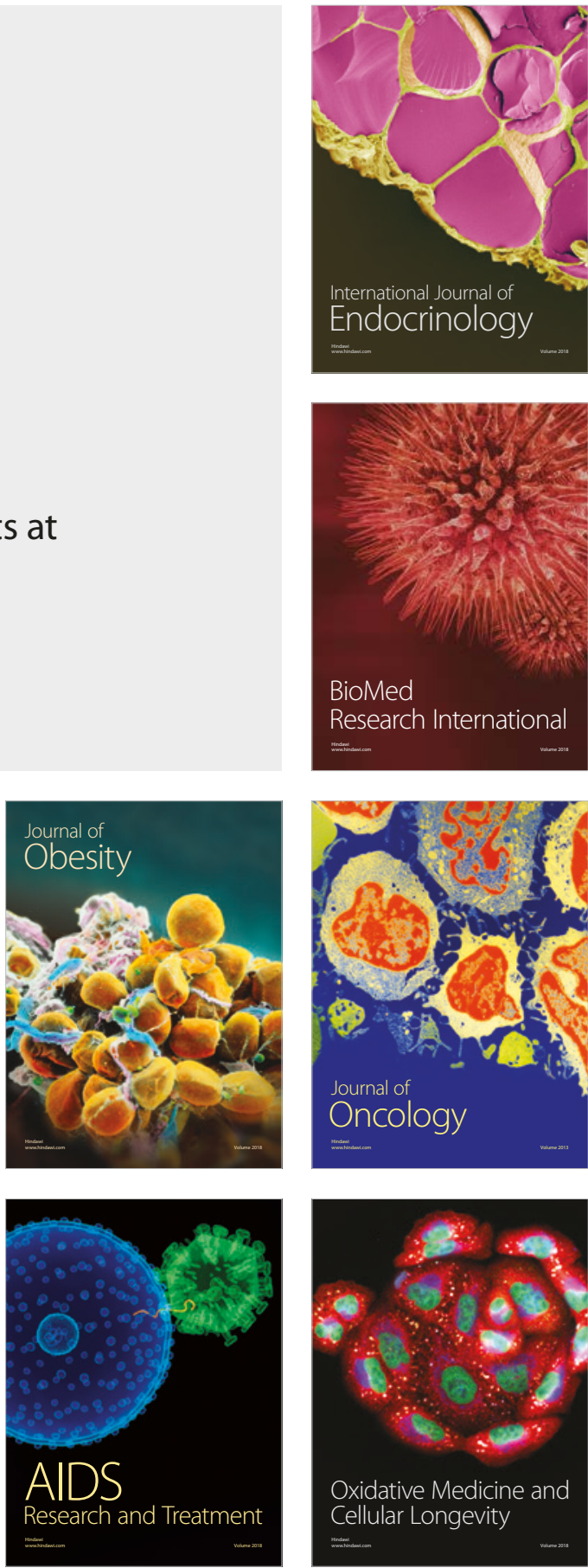